\title{
Adult Blaschkitis With Lichenoid Features and Blood Eosinophilia
}

\author{
Amal Al-Balbeesi ${ }^{1}$ \\ 1. Dermatology, King Khalid University Hospital, Riyadh, SAU
}

Corresponding author: Amal Al-Balbeesi, amalbalbeesi@gmail.com

\begin{abstract}
There have been many reports of congenital and acquired dermatoses that trail Blaschko lines. Lichen striatus is representative of an acquired cutaneous linear inflammatory dermatosis running along the lines of Blaschko, characterized histopathologically by the predominance of lichenoid infiltration. Adult blaschkitis, however, is considered under the same disease spectrum as lichen striatus and is characterized by a spongiotic reaction pattern. Few differences have been recognized between lichen striatus and adult blaschkitis such as age of onset, triggers, distribution, histopathology, and response to treatment.

A case of male patient with adult blaschkitis presenting as unilateral asymptomatic erythematous edematous papules, papulovesicles, and plaques over Blaschko's lines, in which skin biopsy revealed coexistent pathological features of lichen striatus and adult blaschkitis along with blood eosinophilia, has been presented here. This may add to the cumulative evidence on the pathogenesis of adult blaschkitis as an allergic reaction to an unknown inciting allergen.
\end{abstract}

Evidence to consider adult blaschkitis as part of the lichen striatus spectrum is growing. Triggers for such skin reactions remain diverse. This case presentation suggests that adult blaschkitis could be triggered by an allergic response evidenced by the blood eosinophilia when other causes of eosinophilia are excluded.

Review began 07/11/2021 Review ended 07/22/2021 Published 08/03/2021

(๑) Copyright 2021

Al-Balbeesi. This is an open access article distributed under the terms of the Creative Commons Attribution License CC-BY 4.0., which permits unrestricted use, distribution, and reproduction in any medium, provided the original author and source are credited.
Categories: Dermatology, Hematology

Keywords: blaschkitis, mosaic, lichen striatus, eosinophilia, allergic response

\section{Introduction}

Blaschko's lines (BL) are considered systematized cutaneous developmental patterns during embryogenesis that are different from vascular, neural, or lymphatic pathways. The BL were described and drawn by Alfred Blaschko, a German dermatologist, in 1858-1922 [1,2].

There have been many reports of congenital and acquired dermatosis that trail BL. Congenital dermatoses located on BL are incontinentia pigmenti, epidermal nevus, linear nevus sebaceous, and focal dermal hypoplasia (Goltz syndrome) [3-5].

Blaschkolinear acquired inflammatory skin eruption (BLAISE) is a cluster of disorders consisting of various inflammatory skin diseases including lichen striatus (LS), adult blaschkitis (AB), lichen planus (LP), psoriasis, lichen nitidus, atopic dermatitis, lupus erythematosus, and graft-versus-host disease [4]. We report a case of $\mathrm{AB}$ with coexisting pathological features of LS and transient blood eosinophilia, which resolved with the clearing of skin lesions, suggesting an allergic reaction as a possible trigger for the blaschkitis or an associated feature of the skin presentation since other causes of eosinophilia were excluded.

\section{Case Presentation}

A 19-year-old Yemeni male patient (skin type 5) presented to dermatology with a two-week history of a minimally itchy eruption that involved the left side of his trunk, axilla, upper, and lower extremities. Lesions started as deep-seated vesicles on the palm and then spread centripetally to involve the arm, trunk, and lower extremity, as seen in Figure 1. 


\section{Cureus}

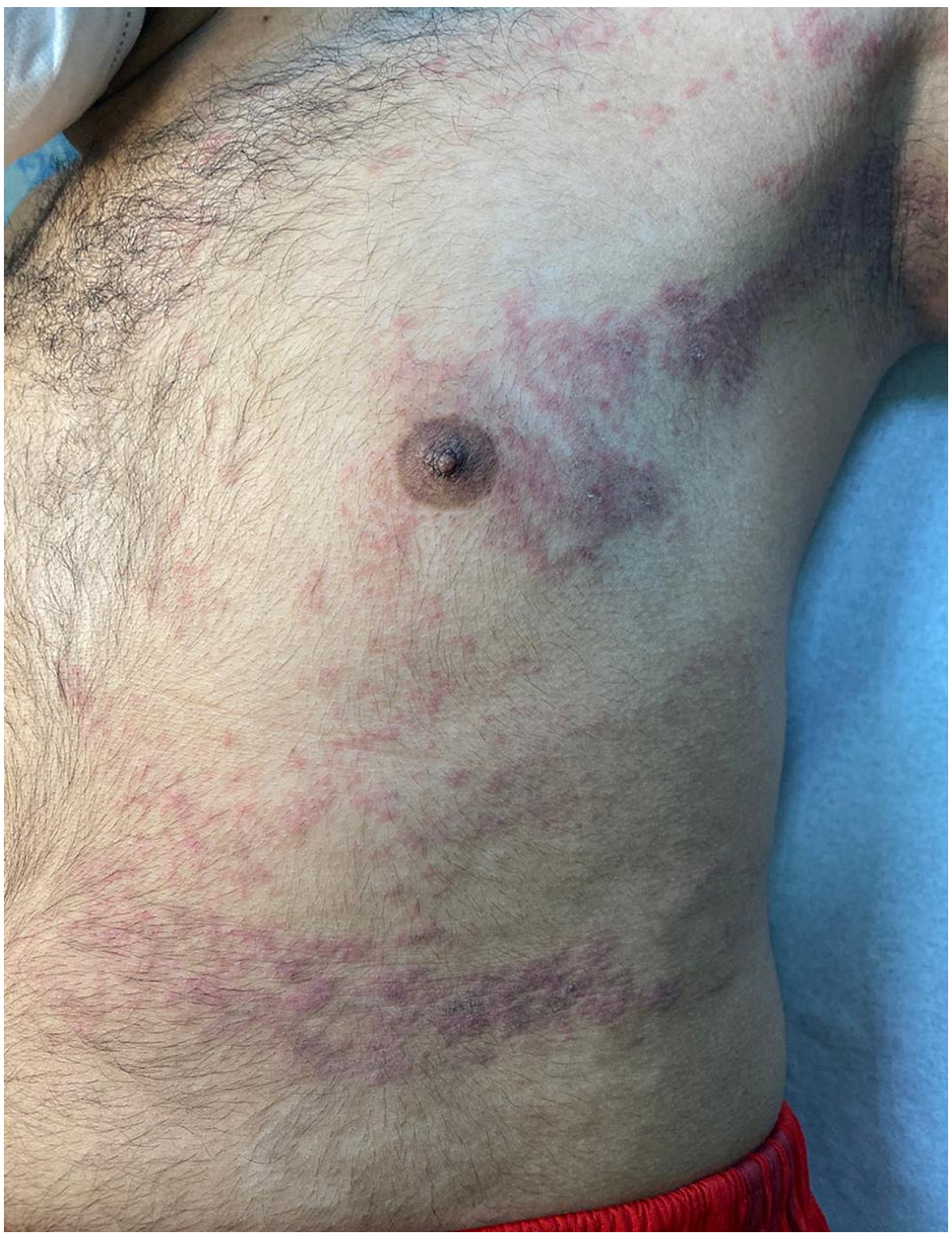

FIGURE 1: Spread of deep-seated vesicles on the arm, trunk, and lower extremity.

There was no personal history of atopy, insect bite, drugs, or recent infection, nor a family history of similar presentation. On examination, unilateral erythematous edematous papules, plaques, and papulo-vesicles over the left side of the body were observed, as seen in Figure 2. 


\section{Cureus}

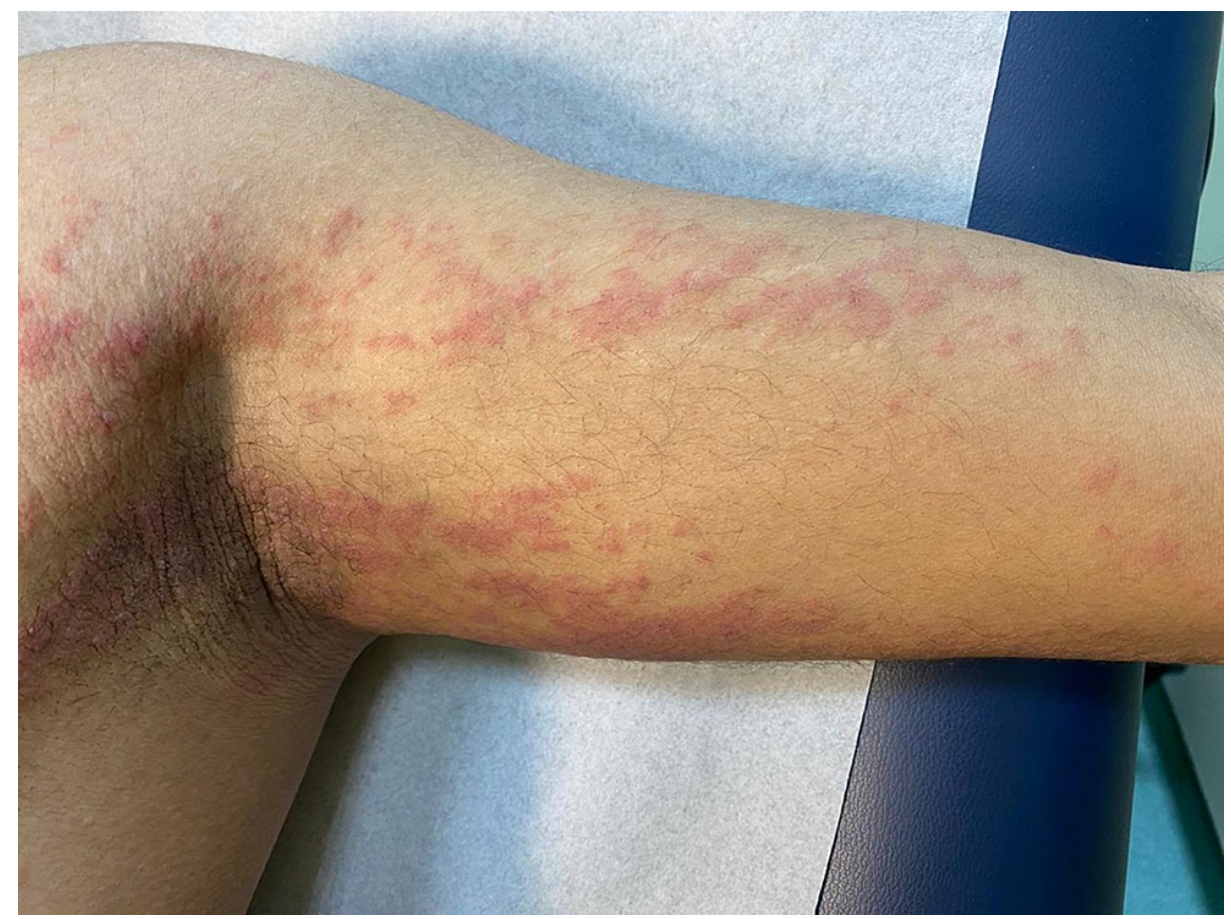

FIGURE 2: Unilateral erythematous edematous papules, plaques, and papulo-vesicles over the left side of the body.

The lesions were following BL as whorls and interrupted lines. Nails and mucous membranes were spared. Complete blood count (CBC) showed hemoglobin and white blood cell count to be within normal limits and eosinophilia of $10.6 \%$. Additionally, renal and liver functions were normal, hepatitis B screening was negative, and antinuclear antibody and stool analysis were negative.

Biopsy showed parakeratosis, epidermal spongiosis, and mild acanthosis with several necrotic keratinocytes mainly at the lower levels of the epidermis. In the dermis, foci of lichenoid infiltrate at the dermo-epidermal junction were found. Lymphocytic infiltrate was also noted around the eccrine ducts. Additionally, scattered eosinophils (seven eosinophils per 10 high-power fields), some extravasated red blood cells, and no evidence of vasculitis were observed, as seen in Figure 3, Figure 4. 


\section{Cureus}

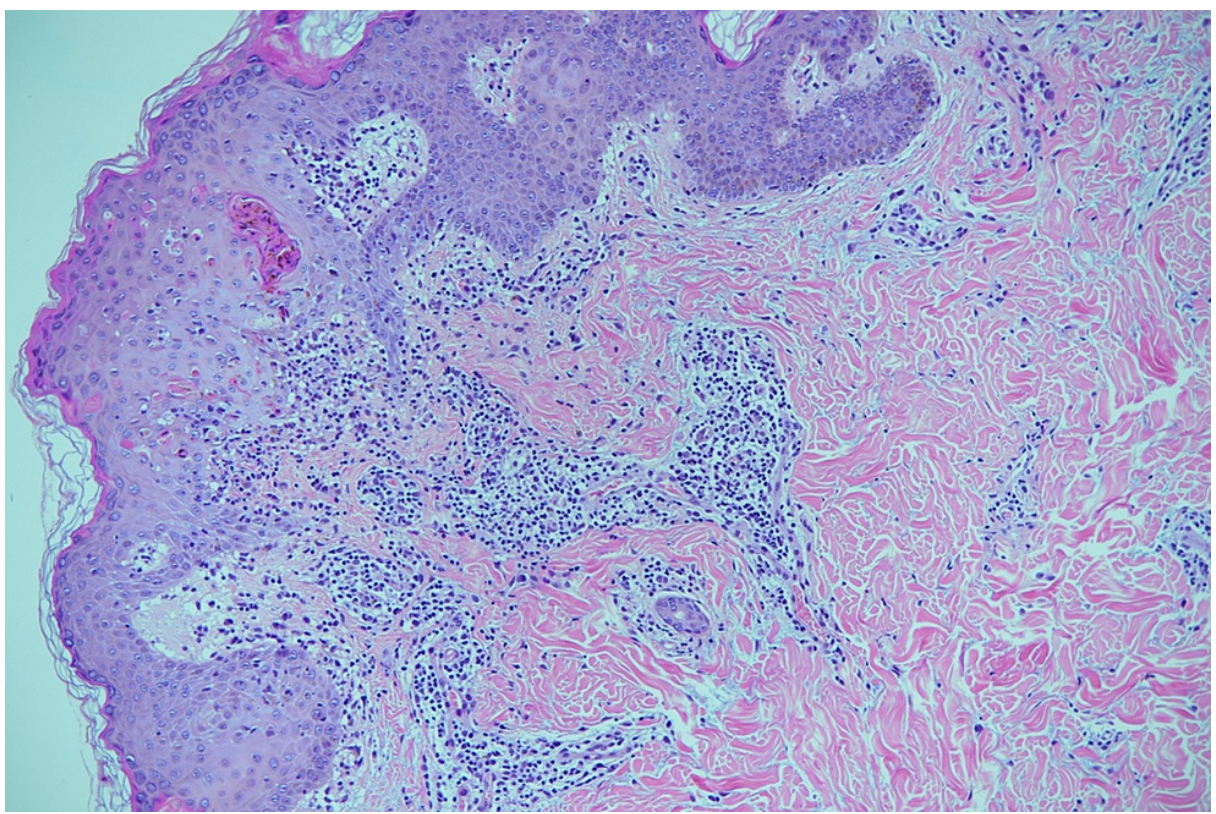

FIGURE 3: Patterns that were consistent with spongiotic and lichenoid reactions with eosinophils.

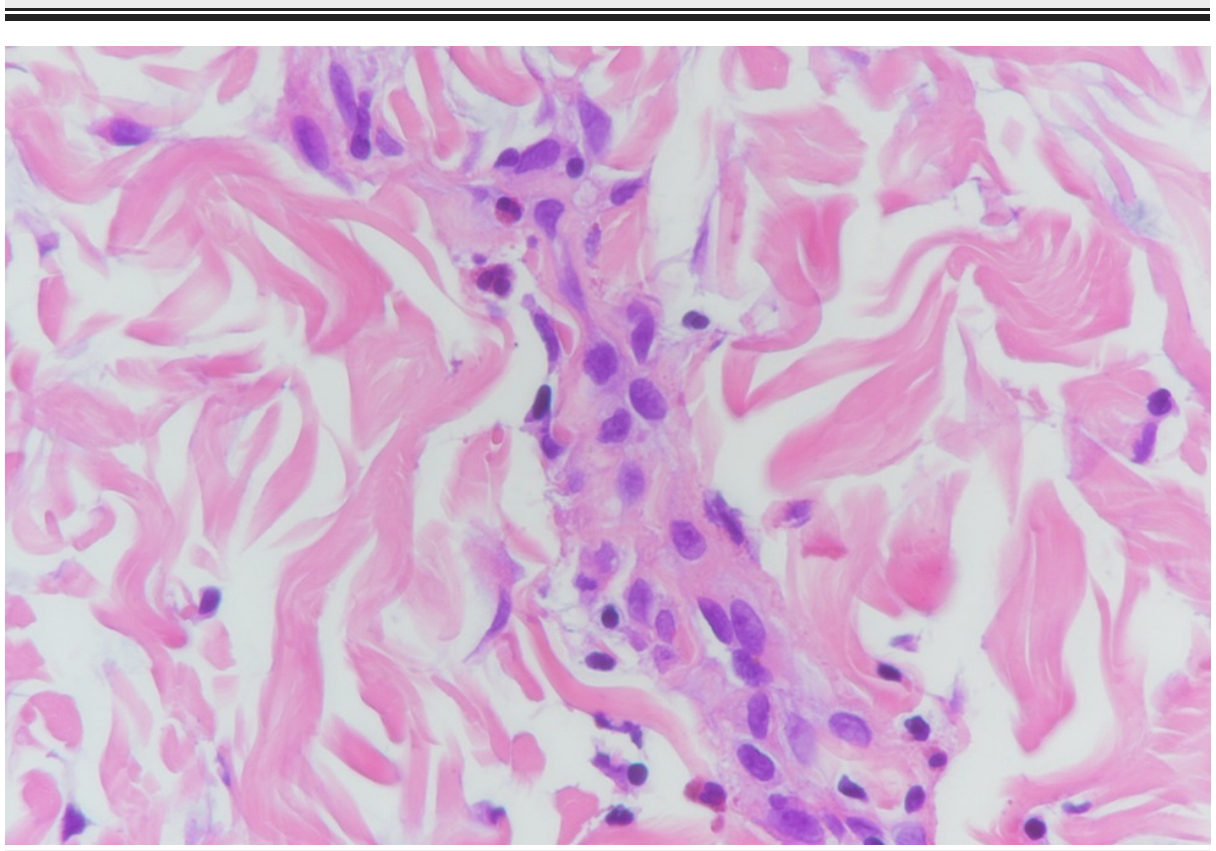

FIGURE 4: Scattered eosinophils (seven eosinophils per 10 high-power fields).

Immunofluorescent studies for IgA, IgG, IgM, C3, and fibrinogen were negative. The lesions resolved with post-inflammatory hyperpigmentation spontaneously within two weeks, as shown in Figure 5. 


\section{Cureus}

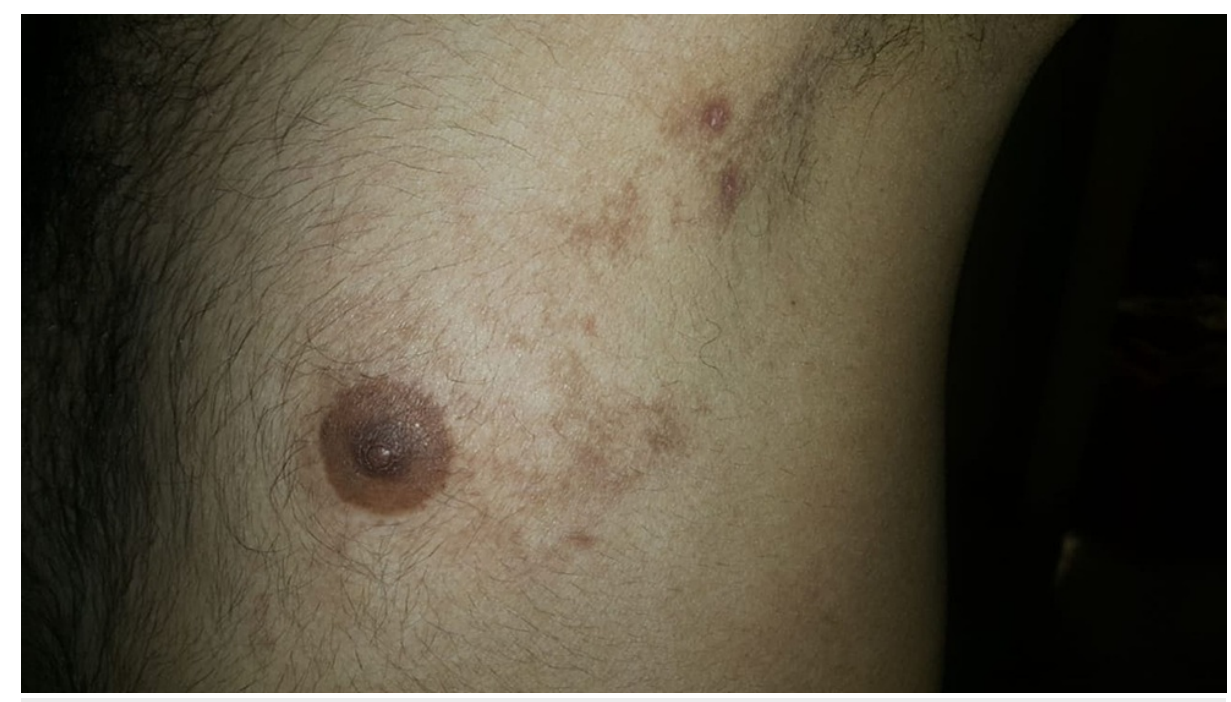

\section{FIGURE 5: The lesions resolved with post-inflammatory}

hyperpigmentation spontaneously within two weeks.

Based on the clinical and histopathological features, the diagnosis of $A B$ with spongiotic dermatitis and the coexistent lichenoid infiltrate with eosinophils was considered. The patient was prescribed topical mometasone cream $0.1 \%$ and a poor compliance was reported but was nonetheless satisfied with the outcome. CBC repeated after 12 weeks revealed no eosinophilia and there was no recurrence of lesions.

\section{Discussion}

$\mathrm{AB}$ is one of the disorders comprising BLAISE along with many others including LS, LP, psoriasis, lichen nitidus, atopic dermatitis, lupus erythematosus, and graft-versus-host disease [4]. AB and LS are still considered under the same disease spectrum; however, few differences have been recognized, such as age of onset, distribution, histopathology, and response to treatment [6-9] (Table 1). 


\section{Cureus}

\begin{tabular}{|c|c|c|}
\hline Features & Adult Blaschkitis & Lichen Striatus \\
\hline Age in years & $18-55$ & $5-15$ \\
\hline Distribution & Multiple sites including extremities and trunk & Unilateral single site (extremities) \\
\hline Clinical & Papulo-vesicles over multiple BL & $\begin{array}{l}\text { Erythematous-brown discrete and coalescing } \\
\text { papules over BL }\end{array}$ \\
\hline Symptoms & Itchy & Sometimes itchy \\
\hline Time of resolution & Few weeks & One year \\
\hline Triggers & $\begin{array}{l}\text { Pregnancy Infections, vaccination, drugs, trauma insect bites, } \\
\text { stem cell transplant }\end{array}$ & Infection, sunburn, contact allergens \\
\hline \multicolumn{3}{|l|}{ Histopathology } \\
\hline Spongiosis & +++ & +++ \\
\hline $\begin{array}{l}\text { Lichenoid } \\
\text { infiltrate }\end{array}$ & + & +++ \\
\hline \multicolumn{3}{|l|}{ Treatment } \\
\hline $\begin{array}{l}\text { Response to } \\
\text { treatment }\end{array}$ & Favorable & Less favorable \\
\hline $\begin{array}{l}\text { Spontaneous } \\
\text { resolution }\end{array}$ & +++ & +++ \\
\hline Recurrence & Common & Rare \\
\hline
\end{tabular}

TABLE 1: Differences between adult blaschkitis and lichen striatus[6-24].

+++, frequently reported; +, less commonly reported.

BL, Blaschko's lines

LS is representative of acquired cutaneous linear inflammatory dermatosis running along the BL, usually in a unilateral fashion and mainly over extremities [6-9]. LS primarily occurs in children between 5 and 15 years of age. Involvement of the nail in LS is rare and may occur before, concurrently, or after the skin lesion [25]. The affected nail may present as pitting, longitudinal ridging, fissuring, splitting, fraying, striate or punctate leukonychia, overcurving, thinning or thickening of the nail plate, irregular transverse grooves, onycholysis, subungual hyperkeratosis, and nail loss. The presentations may only include the lateral or medial portion of the nail plate of only one digit [25]. The case series reported by Lunge et al. showed resolution of LS lesions within three months of tazarotene use with minimal discomfort due to irritation [26]. Topical steroids had been used for itchy LS lesions with inconsistent results [26]. However, calcineurin inhibitors and calcipotriol with topical or intralesional steroids have been used successfully [26]. Spontaneous resolution of LS had been reported within 3-12 months [26].

$\mathrm{AB}$ affects adults between 15 and 88 years of age and is characterized histopathologically by spongiotic dermatitis primarily as compared to LS, which shares similar pathological features but prominent lichenoid infiltrate [6-10]. Multiple BL are affected over trunk and extremities. Unlike LS, AB is recurrent, a feature that is reported rarely in LS. It is suggested that LS and AB are triggered by many extrinsic stimuli such as pregnancy [11], infection [12-14], vaccination [15-17], drugs [18-21], trauma [22], insect bite [23], autoimmune phenomena [7,10], and stem cell transplant [24]. These stimuli bring about somatic mutations in keratinocytes resulting in a T-cell immune hypersensitivity response directed against keratinocytes exhibiting mosaicism along BL.

We report here a case of $\mathrm{AB}$ with spongiotic dermatitis and coexistent lichenoid infiltrate with concurrent tissue and blood eosinophilia. Persistent eosinophilia for more than three months can fall into three categories: reactive, clonal, or syndromic such as hypereosinophilic syndrome [27]. Many diseases including allergic conditions such as atopic dermatitis, drugs, infections, autoimmune, and malignancy could lead to eosinophilia [27]. Clinical history is the most important guide to the cause. The lack of any specific finding that points to the cause of eosinophilia in this case including atopic dermatitis made $A B$ the probable cause of eosinophilia. Eosinophils are rare in LP and its variants; however, some cases of hypertrophic LP have 
eosinophils in the absence of drug history [28]. Hypertrophic LP is to be included in the differential diagnosis of lichenoid dermatitis with eosinophils [28]. Nonetheless, the lack of pruritis, the morphology of the lesions, the short duration, and histopathology are against LP.

We suggest that this patient presentation could be triggered by an allergic reaction to an unknown inciting agent or a true additional feature of $\mathrm{AB}$ and to include it in the differential diagnosis of lichenoid dermatitis with eosinophils. Performing a full blood count of such cases may shed light on the etiology and the features of this disease entity.

\section{Conclusions}

In conclusion, $\mathrm{AB}$, a diagnosis subjected to major scrutiny, shares many features with LS and LP. The age of onset, the short duration of the disease, and the histopathological features, all collectively point in favor of $A B$. Features against LP include the lack of intense pruritis and the clinicopathological features. $A B$ triggers can be diverse; an allergic hypersensitivity reaction could be added to the list and we suggest obtaining eosinophils count to try to understand the triggering factors for such a presentation. Skin eruption resolves spontaneously without treatment with post-inflammatory hyperpigmentation as with our patient and other reported cases. More case reports are needed to understand the triggers of such a presentation to improve our true understanding of the pathogenesis of $\mathrm{AB}$ and its associated features.

\section{Additional Information \\ Disclosures}

Human subjects: Consent was obtained or waived by all participants in this study. Conflicts of interest: In compliance with the ICMJE uniform disclosure form, all authors declare the following: Payment/services info: All authors have declared that no financial support was received from any organization for the submitted work. Financial relationships: All authors have declared that they have no financial relationships at present or within the previous three years with any organizations that might have an interest in the submitted work. Other relationships: All authors have declared that there are no other relationships or activities that could appear to have influenced the submitted work.

\section{References}

1. Jackson R: The lines of Blaschko: a review and reconsideration: observations of the cause of certain unusual linear conditions of the skin. Br J Dermatol. 1976, 95:349-60. 10.1111/j.1365-2133.1976.tb00835

2. Bolognia JL, Orlow SJ, Glick SA: Lines of Blaschko. J Am Acad Dermatol. 1994, 31:157-90; quiz 190-2. 10.1016/s0190-9622(94)70143-1

3. Grosshans EM: Acquired blaschkolinear dermatoses. Am J Med Genet. 1999, 85:334-7.

4. Müller CS, Schmaltz R, Vogt T, Pföhler C: Lichen striatus and blaschkitis: reappraisal of the concept of blaschkolinear dermatoses. Br J Dermatol. 2011, 164:257-62. 10.1111/j.1365-2133.2010.10053.x

5. Suárez-Peñaranda JM, Figueroa O, Rodríguez-Blanco I, Aliste C, Casas L: Unusual interface dermatoses distributed along Blaschko's lines in adult patients. Am J Dermatopathol. 2017, 39:144-9. 10.1097/DAD.0000000000000689

6. Baek YS, Seo JY, Seo SH, et al.: Adult-onset lichen striatus versus adult blaschkitis: a clinicopathological review of 40 cases of acquired blaschkolinear inflammatory dermatosis. Eur J Dermatol. 2019, 29:281-6. 10.1684/ejd.2019.3556

7. Hofer T: Lichen striatus in adults or 'adult blaschkitis'?. There is no need for a new naming . Dermatology. 2003, 207:89-92. 10.1159/000070955

8. Zhang Y, McNutt NS: Lichen striatus. Histological, immunohistochemical, and ultrastructural study of 37 cases. J Cutan Pathol. 2001, 28:65-71. 10.1034/j.1600-0560.2001.280202.x

9. Charles CR, Johnson BL, Robinson TA: Lichen striatus: a clinical, histologic and electron microscopic study of an unusual case. J Cutan Pathol. 1974, 1:265-74. 10.1111/j.1600-0560.1974.tb00638.x

10. Patrizi A, Neri I, Fiorentini C, Bonci A, Ricci G: Lichen striatus: clinical and laboratory features of 115 children. Pediatr Dermatol. 2004, 21:197-204. 10.1111/j.0736-8046.2004. 21302.x

11. Brennand S, Khan S, Chong AH: Lichen striatus in a pregnant woman . Australas J Dermatol. 2005, 46:184-6. 10.1111/j.1440-0960.2005.00176.x

12. Ishikawa M, Ohashi T, Yamamoto T: Lichen striatus following influenza infection. J Dermatol. 2014, 41:1133-4. 10.1111/1346-8138.12684

13. Richarz NA, Fusta-Novell X, Fatsini-Blanch V, Fortuny C, Gonzalez-Ensenat MA, Vicente A: Lichen striatus following scarlet fever in a 3-year-old female patient. Int J Dermatol. 2018, 57:1118-9. 10.1111/ijd.13995

14. Hafner C, Landthaler M, Vogt T: Lichen striatus (blaschkitis) following varicella infection. J Eur Acad Dermatol Venereol. 2006, 20:1345-7. 10.1111/j.1468-3083.2006.01691.x

15. Karakaş M, Durdu M, Uzun S, Karakaş P, Tuncer I, Cevlik F: Lichen striatus following HBV vaccination. Dermatol. 2005, 32:506-8. 10.1111/j.1346-8138.2005.tb00791.x

16. Karouni M, Kurban M, Abbas O: Lichen striatus following yellow fever vaccination in an adult woman . Clin Exp Dermatol. 2017, 42:823-4. 10.1111/ced.13167

17. Hwang SM, Ahn SK, Lee SH, Choi EH: Lichen striatus following BCG vaccination. Clin Exp Dermatol. 1996, 21:393-4. 10.1111/j.1365-2230.1996.tb00137.x

18. Mask-Bull L, Vangipuram R, Carroll BJ, Tarbox MB: Lichen striatus after interferon therapy. JAAD Case Rep. 2015, 1:254-6. 10.1016/j.jdcr.2015.05.002

19. Brinkmeier T, Herbst RA, Schaller J, et al.: Drug-induced blaschkitis. Acta Derm Venereol. 2004, 84:314-5. 


\section{Cureus}

10.1080/00015550410025282

20. Lora V, Kanitakis J, Latini A, Cota C: Lichen striatus associated with etanercept treatment of rheumatoid arthritis. J Am Acad Dermatol. 2014, 70:e90-2. 10.1016/j.jaad.2013.11.039

21. Monteagudo B, Cabanillas Ml, Suarez-Amor O, Ramirez-Santos A, Alvarez JC, de Las Heras C: [Adult blaschkitis (lichen striatus) in a patient treated with adalimumab]. Actas Dermosifiliogr. 2010, 101:891-2.

22. Shepherd V, Lun K, Strutton G: Lichen striatus in an adult following trauma. Australas J Dermatol. 2005, 46:25-8. 10.1111/j.1440-0960.2005.00132.x

23. Unal E, Balta I, Bozkurt O: Lichen striatus: after a bite by bumblebee . Cutan Ocul Toxicol. 2015, 34:171-2. 10.3109/15569527.2014.910804

24. Mun JH, Park HJ, Kim HS, Kim SH, Ko HC, Kim BS, Kim MB: Lichen striatus occurring after allogenic peripheral blood stem cell transplantation in an adult with aplastic anemia. Ann Dermatol. 2012, 24:87-9. 10.5021/ad.2012.24.1.87

25. Leung AK, Leong KF, Barankin B: Lichen striatus with nail involvement in a 6-year-old boy . Case Rep Pediatr. 2020, 2020:1494760. 10.1155/2020/1494760

26. Lunge S, Vupperla D, Patil V: Linear lichen striatus - Response to topical tazarotene - A case series and review. IP Indian J Clin Exp Dermatol. 2019, 5:228-31. 10.18231/j.ijced.2019.049

27. Sims H, Erber WN: Investigation of an incidental finding of eosinophilia. BMJ. 2011, 342:d2670. 10.1136/bmj.d2670

28. Alomari A, McNiff JM: The significance of eosinophils in hypertrophic lichen planus . J Cutan Pathol. 2014, 41:347-52. 10.1111/cup.12275 